\title{
Magnetic field of the Hot Earth. Reversals, magnetic anomalies and mechanism of the magnetic poles drift.
}

\author{
Vladimir Kuznetsov* \\ Institute of Cosmophysical Research and radio wave propagation FEB RAS, \\ 684034, Kamchatka, Paratunka, Mirnaya str., 7, Russia
}

\begin{abstract}
Principally new model of the magnetic field of the Hot Earth is proposed. Unlike the commonly accepted approach which considers that the Earth's temperature doesn't increase because heat released under selfgravitation is removed through radiation our model assumes that early substance of the Earth heated up to $30000 \mathrm{~K}$ was a superheated and overcompressed vapour.

Cooling the Earth substance was condensing. The system was expanding adiabatically that governed the character of the Earth enlargement. This scheme origins from the phase transition (PT) of condensation-evaporation under the benefit of condensation. PT provides the heat, geodynamics of expansion and the Earth's magnetic field (EMF).

The high temperature of the substance causes its thermoionization, whereas PT operation relating to mass transfer initiates charges separation and generation of the double electric layer (DEL). A diurnal rotation of DEL induces a weak initial EMF which enhances then at the expense of the Hall dynamo (Hall current) inside PT area. The benefit of evaporation causes the Earth compression and reversal of the EMF polarity.

The approach we develop provides an insight into features of the magnetic field of the planets and satellites at the Sun system.
\end{abstract}

Key words: The Hot Expanding Earth, phase transition and charges separation in the Flayer, DEL generation, daily rotation of DEL, weak magnetic field and its amplification by the Hall dynamo in the F-layer.

\section{Introduction.}

At the conference "The inner core - 2000" in Moscow the leading Russian magnitilogist Prof. Petrova declared that the problem of the geomagnetic field (GMF) being the insight into the mechanism of its generation source. It is obvious that the heat convection is responsible for the generation of the GMF at the liquid iron core.

By the data of Prof. Dario Alfe and his colleagues (ref. in [1]) who were the first to calculate the Earth heat conductivity using the density functional theory, the heat convection of the Earth core is not able to provide the source of the GMF. As was revealed the Earth inner core started its formation rather $1 \mathrm{Ga}$ ago than 3.4 that followed from earlier research of magnetic minerals in ancient rocks.

The facts reported here suggest that geomagnetism theory is far from its full acceptance. The clause stating that the core of the Earth is a source of its magnetic field isn't validated.

* e-mail: vvkuz38@mail.ru 
The sudden increase of the North magnetic pole (NMP) drift velocity during the 1990s has plagued the magnetologists [2] who don't consider the NMP in the frames of the magnetic dynamo as a magnetic field source at the liquid iron core. Up to now the magnetic field of other planets and satellites is assumed to be identical to the Earth's one. The following problems such as why the analysis of the EMF morphology points to its generation source at the border of the inner core $(\mathrm{G})$ and why its source nature is unknown, why superchrones and superplumes coincide in time are unsolved.

It is not clear now why the EMF reverses its polarity and the nature of excursions, jerks and, in fact, the drift of the NMP, which is not chaotic, but follows an unknown pattern retain uncertain. As we know the magnetic field of the Moon and Mars disappeared and there is still a dipole field at Mercury. It was Einstein who considered the problem of EMF very important and he attributed it to the five most important tasks in physics. The source of EMF generation is not interpreted on the concept of the theory of the iron core and heat convection for source of the EMF generation.

As in my report I briefly outline the main points of our EMF model then I propose here our concept of the Solar system and our model of the hot expanding Earth.

\section{Solar system.}

\subsection{Density.}
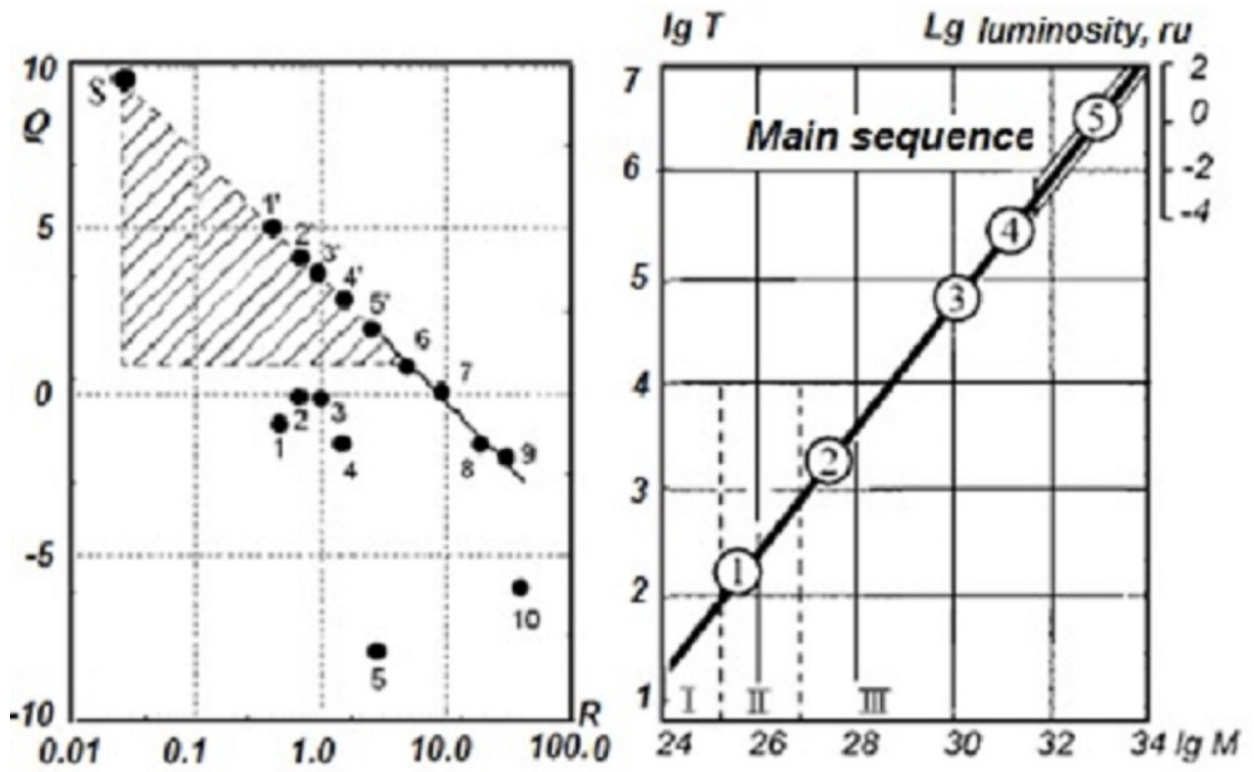

Fig.1. The surface density for the planets of the Solar system $(\lg -\lg )$. The mass of the shaded triangle is equal to the mass of the Sun. Numbers - planet numbers from Mercury (1) up to Pluto (10), 5 Asteroid belt [3].

Fig. 2. The formation temperature $T$ as a function of mass $M$ : Sun - 5, Brown Dwarf - 4, Jupiter - 3, Earth - 2 and Moon - 1. The right upper corner - the luminosity dependence for the Main Sequence stars (MS, in rel.un.) on their mass. Objects of I, II, III - classes are included into the Solar system [4].

As the structure consisting of the Sun and its planets can be represented as twodimensional it makes sense to talk not about its surface density. It is logical to assume that each planet was formed from the substance that was concentrated inside the ring, limited by its own radius of the $n$-th planet and the radius of the inner planet relative to it, $n-1$, where $\mathrm{n}$ is the serial number of the planet (asteroid belt we also consider to be a planet). Dividing 
the mass of the planet by the ring's area, we get the value of the surface density $\rho=$ $M_{\mathrm{n}} / \pi\left(R_{\mathrm{n}}^{2}-R^{2}{ }_{\mathrm{n}-1}\right)$.

\subsection{Disc compression and Jeans criterion.}

From Jeans criterion it follows that the substance concentration is more than a crucial density $\rho_{C}$ and that gravity and the inner compression are accurately balanced. The density of the substance with mass $M$ and temperature $T$ [3]: $m_{C}=10^{3}\left[T^{3} /(M / M \odot)\right] \mathrm{cm}^{-3}$. Valid for Jupiter, Saturn, Uranus and Neptune the self-gravity condition does not apply to the planets of the Earth group because their mass is less than the crucial Jeans one: $M_{\mathrm{Jn}} / M_{\mathrm{n}}$ for the Earth and Mars is $10^{4} ; 10^{5}$ and it is 0.3 for Jupiter. For Jupiter, Saturn, Uranus and Neptune the dependence of the surface density $\rho$ on their distance to the Sun $R$ is shown in Fig.1: $\rho=A$ $R^{- \text {n }}$, here $n \approx 3.55$ and $A$ is the constant. This dependence is interrupted in the area of the asteroid belt (5'), where at the time of formation of the Sun and planets there was a break, bifurcation in the chain of fragmentation processes of protosolar disc into separate rings. The formation of Jupiter, Saturn, Uranus and Neptune out of the rings separated from the disc continued, and the substance of each ring assembled into a large planet with the satellites system similar to that of the Sun. During bifurcation the planets of the Earth group had formed only nucleus of future planets and all the remaining mass of the protosolar disk (hydrogen, mainly) spent on the formation of the Sun mass $M_{\odot}$ (the shaded triangle at Fig.2).

\subsection{Temperature of the Sun, planets and satellites.}

The correlation $[3,4]$ for the Main sequence $\left(T \sim M^{2 / 3}\right)$ follows from the comparison of two curves at Chandrasekar book, they are the $T$-dependence of luminosity (in rel. un.) and luminosity related to the star mass $M$ (Fig. 2, right top). Fig.2 suggests that bodies of the Sun system are divisible into three classes, they are $G M / R<U_{\mathrm{m}}, U_{\mathrm{m}}<G M / R<U_{\mathrm{e}}$ and $G M / R>U_{\mathrm{e}}$, here $U$ - heat of phase transition, they are melting $(m)$ and evaporation $(e)$. Small satellites and asteroids are among the first class (Fig.2), large satellites and small planets (less than Venus) are at the second one and planets of mass more than Venus enter the third one.

\section{Physics of hot expanding Earth.}

\subsection{Hot Earth.}

What is the difference between hot and cold models of the Earth? Here we follow Magnitsky [5]. Dividing the gravitational energy of the Earth $E=3 / 5 G M^{2} / R=2.25 \times 10^{39}$ erg (here: $G$ is the gravitational constant, $M$ - the Earth's mass and $R$ - its radius) by heat capacity of the Earth's substance $\left(c_{p}=0.3 \mathrm{cal} / \mathrm{g} \cdot \mathrm{grad}\right)$ and its mass we see the temperature of the Earth's matter of about $30000 \mathrm{~K}$. As Magnitsky discuss: "geophysicists do not know how to use this estimate. The temperature of $30000 \mathrm{~K}$ seems to them to be ridiculous because it implies at least melting of the Earth that contradicts its inner arrangement. The thickness of the Earth's mantle is about 3 thousand kilometers. As the Earth's lifetime $(t)$ is $4.5 \mathrm{Ga}\left(t=1.5 \times 10^{17} \mathrm{~s}\right)$ then if the whole Earth was molten, its substance would crystallize to a depth $(\delta)$ not exceeding $400-600 \mathrm{~km}$ during this time $\left(\delta \approx(\zeta t)^{1 / 2}\right.$, here $\zeta$ thermal conductivity of the Earth's matter $\left.\left(\approx 10^{-2} \mathrm{~cm}^{2} / \mathrm{s}\right)\right]$. This argument almost stopped trying to develop a model of "hot" Earth and the " cold" Earth model is the only model accepted now. 


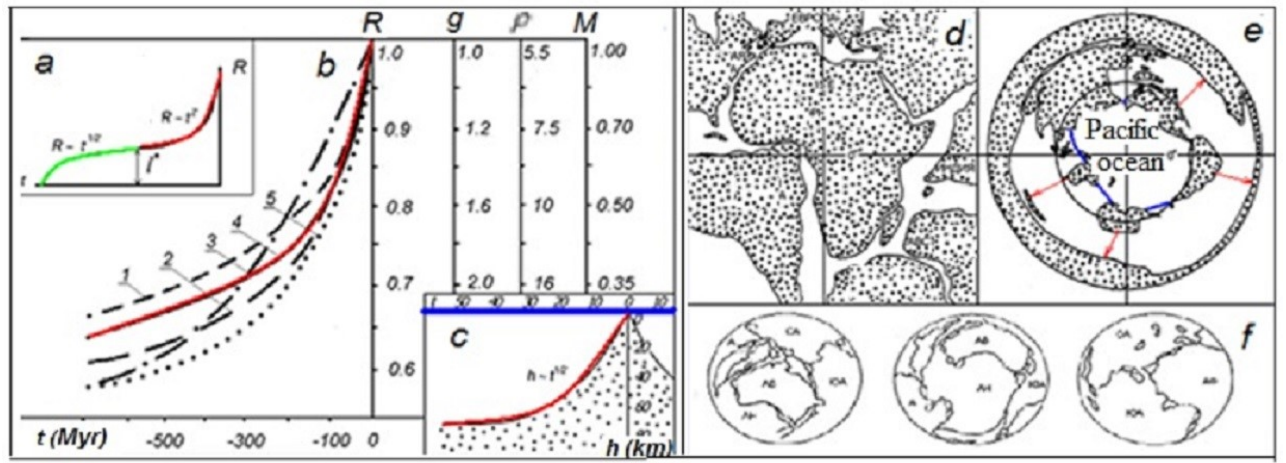

Fig. 3. Time-variation of the Earth's radius $R$ : during the entire period of its development $-a$, over the past $500 \mathrm{Myr}-b$. Here $R(t), g(t), \rho(t): 1$ - according to Carey, 2 - Hildenberg, 3 - Owen, 4 - our model [3]. Increase of the ocean mass $M(t)-5$. Lithosphere thickness $h(\mathrm{~km})$ on the distance $(t$ in million years) from the rift axis $[3,4]-c$. The location of the continents relative to Africa- $d$. Equatorial projection of the perimeter of the Pacific ocean and the direction of the continents drift [6] $-e$. The location of the continents on the sphere of the Earth's outer core [7]- $f$.

The thickness of the Earth's mantle is about 3 thousand kilometers. As the Earth's lifetime $(t)$ is $4.5 \mathrm{Ga}\left(t=1.5 \times 10^{17} \mathrm{~s}\right)$ then if the whole Earth was molten, its substance would crystallize to a depth $(\delta)$ not exceeding $400-600 \mathrm{~km}$ during this time $\left(\delta \approx(\zeta t)^{1 / 2}\right.$, here $\zeta$ thermal conductivity of the Earth's matter $\left.\left(\approx 10^{-2} \mathrm{~cm}^{2} / \mathrm{s}\right)\right]$. This argument almost stopped trying to develop a model of "hot" Earth and the " cold" Earth model is the only model accepted now.

\subsection{Radius of the Earth.}

After its formation the hot Earth began to cool. At the first stage, its outer shell, a prototype of the Earth's continental, granite crust, crystallized. The proto-Earth, gaseous (plasma) after its formation, was condensing, forming liquid substance of the outer core, and then it was crystallizing, forming a mantle. Thus, two exothermic phase transitions, condensation and crystallization, were directly involved into the evolution of our planet. At the earliest stage of the Earth's evolution, the rate of cooling determined the crystallization. This process was controlled by conductive heat transfer - thermal diffusivity: $\zeta=\lambda / \rho c_{p}$, here $\rho$ is the density and $\lambda$ is the coefficient of thermal conductivity. For the Earth substance $\zeta=0.1-0.01\left(\mathrm{~cm}^{2} / \mathrm{s}\right)$. The mantle was building was very slowly: $l \sim(\zeta t)^{1 / 2}, t-$ time of evolution, up to the thickness which was sufficient for convection $\left(l \geq l^{*}\right)$ to occur in the outer core and in the plastic part of the mantle and the convective heat transfer, more efficient than the conductive one operated.

\subsection{Convection in the core and mantle.}

It is known that convection in a layer of thickness $l$ between two planes maintained at a constant temperature drop $\Delta T$ occurs when the Rayleigh number $\operatorname{Ra}>1710: R a=(g \beta$ $\left.l^{3} \Delta T\right) /(\eta \zeta)$. Substituting gravitational acceleration $g$, the volume expansion coefficient $\beta$, the kinematic viscosity $\eta$, the thermal diffusivity coefficient $\zeta$ for $\Delta T=10^{4} \mathrm{~K}$ which are characteristic for the plastic part of the mantle, we get $l^{*}$ about $300 \mathrm{~km}$.

Chandrasekchar (references in [4]) showed that if the Rayleigh numbers are large (at the convection in the core and in the mantle) the product of Reynolds [3]: $\operatorname{Re}=\left(v_{k} l\right) / \eta$ and Prandtl $\operatorname{Pr}=\eta / \zeta$ numbers is proportional to the square root of the Rayleigh number: $\operatorname{Re} x$ 
$\operatorname{Pr} \sim R a^{1 / 2}$. As the Rayleigh number $R a \sim l^{3}$ then the dependence between the characteristic size $l$, the convection rate in the plastic mantle $v_{k}\left(v_{k}=l / t\right)$ and the characteristic time $t: v_{k} l$ $\sim l^{3 / 2}$ or $l^{2} / t \sim l^{3 / 2}$. Replacing $l$ with $\Delta R$ we obtain: $\Delta R \sim t^{2}$ instead of $\Delta R \sim(t)^{1 / 2}$ at the start of the Earth evolution (the insert at the upper left corner of Fig. 3-a).

\subsection{Meservey paradox.}

As Meservey [6] showed that the expanding Earth is unique to agree the modern topology of the continents and the oceans with paleomagnetic data, such as the drift of magnetic poles and magnetic stripe anomalies in the oceans. Continents association into Gondwana, in which Africa, South and North America, Australia and Antarctica form a single continent is impossible over the globe with a radius equal to the present one.

Meservey called attention to the fact that 200 million years ago the continents located along the Pacific shores couldn't be at the distances from each other more than the present ones (Fig. 3-d). To form Gondwana (Fig. 3-d-e), these continents would have to move along the directions shown in Fig.3-e, doubling the surface area of the Pacific ocean, that is contrary to paleoreconstructions. As shown (Gorai, 1984; Kuznetsov, 1990; Carey, 1976; Vogel, 1989; references in [3]), continents can be laid one to another with virtually no gaps on the ball with the radius equal to that of the Earth's outer core (Fig. 3-f). To support this fact, Dr. Vogel made a transparent globe, inside which the continents the same as on the globe were located on the ball, equivalent to the outer core [6]. Vogel's globe shows how the position of the continents had been varying from the time of the Earth's formation up to the modern one.

\subsection{Heat flow.}

Assuming that all mantle matter condensed and crystallized, and the substance of the outer core condensed [3] we estimate the value of energy $E_{U}$ released by the Earth during its evolution ( $t=4.5$ billion years) by means of a phase transition. We take here that almost all the Earth's substance (except that of the inner core, which is only a small part, of about $1-2 \%$ ) underwent a phase transition. $E_{U}=6 \times 10^{38} \mathrm{erg}$, that is about $1 / 3-1 / 4$ of the Earth's total gravitational energy. Almost all $E_{U}$ should supply the heat flow $Q$. Estimating the value of $Q$, averaged throughout the Earth's evolution $t: Q=E_{U} / t=10^{29} \mathrm{erg} /$ year we get $Q$ of about an order of magnitude greater than currently observed $\left(1.5 \times 10^{-6} \mathrm{cal} / \mathrm{cm}^{2} \mathrm{~s} \approx 10^{28}\right.$ erg/year), but this discrepancy should not be particularly confusing, since the temperature gradient $d T / d p$ and, consequently, $Q$, are known to be significantly higher at earlier geological epochs. (here the heat flows at the continents and the oceans are obviously equal).

Here are some estimates:

- the annual power of the Earth's heat flow is approximately $3 \times 10^{13} \mathrm{~W}$

- the power of the Earth's magnetic field is $\approx 10^{12} \mathrm{~W}$,

- the power released during earthquakes and volcanic eruptions $\approx 3 \times 10^{11} \mathrm{~W}$.

The mechanical efficiency of " Earth heat machine " is seen to be small (about a percent), the converting efficiency of energy into the magnetic field is 3 times higher, etc

\section{The magnetic field generation in the context of the hot Earth model.}

\subsection{Location and size of the geomagnetic field generation source.}

In the model of geodynamo it is assumed that the dipole source is the geomagnetic field placed in the outer core, nearby its border with the mantle or on its border. Not discussed in Geomagnetism the location of the generation source, is however, known after 
attempts to determine the source size of (the depth of its location in relation to the Earth's radius $R$ ) [3]. The depth of the source was estimated from the structure of the geomagnetic field in several ways [8]. It was shown that the source is located at the boundary of the inner core or at its F-layer.

\subsection{The importance of the new model of the geomagnetic field generation.}

Despite its urgency the problem of Geomagnetism is still far from being solved. For more than for 50 years the only model of the geomagnetic field generation - the magnetic dynamo which is based on the model of the cold Earth, iron core and the uncertain source of the Earth internal energy has dominated. Parkinson [9] considers that: "currently, researchers discuss three possible sources of energy: convective heat exchange due to either the initial heat of the core or the energy of radioactivity; due to convection caused by physical or chemical processes including secular variations at one of the outer core boundaries; due to the liquid flux driven by the gravitational moments generated by the Sun and the Moon relative to the Earth." In the author's opinion, no one has yet proposed a solution that could account for the actual situation inside the Earth's core [9]. Such phenomena of Geomagnetism as reversals and excursions, Western drift and drift of magnetic poles, etc. are far from their interpretation. Naturally, in the frames of modern concepts it is impossible to create a model that is adequate to the existing geomagnetic field and it is necessary to create a principally new model of the Earth capable to explain all past, modern and future phenomena.

\subsection{Seismic waves velocity in the G-core.}

As shown the velocity of P- and S- waves in the inner core do not change as they approach the center of the Earth, i.e., otherwise, the velocities of P- and S-waves in the inner core do not depend on pressure increasing (as it approaches the center of the Earth) about three times. The distribution of P-waves inside the Earth's core (red line) and iron shown in Fig. 4-A, a suggests that the core of the Earth is not iron. This conclusion has been repeatedly supported by other examples. It is reasonable to suppose that the Earth's core as well as its mantle consists mainly of silicon oxide, water and hydrogen.

\subsection{Velocity of seismic waves in the F-layer.}

The velocitiy distribution of sound (P-waves) in the F-layer adjacent to the inner core is ambiguous and there are several interpretations. Now the authors tend to the interpretation of Jeffries, according to which at the boundary of the F-layer with the E-core the sound speed drops from 10.4 to $9.5 \mathrm{~km} / \mathrm{s}$, and then it increases up to $11.2 \mathrm{~km} / \mathrm{s}$ at the boundary of the F-layer and the inner G-core (Fig. 4-A, a). Note that the behavior of the Pwave velocity inside the F-layer resembles the distribution of the sound velocity in a twophase system [10] near the point of the phase transition. The sound velocity at the liquidvapor boundary is always less than in liquid (phase 1) and in gas (phase 2).

\subsection{Electrical conductivity of the F-layer.}

The Earth's core is considered to consist of iron, which is liquid in the outer core and solid in the inner core and therefore there should be a jump in conductivity of about some orders in magnitude at the core-mantle boundary. Detected by electromagnetic sensing methods such a jump would confirm the model of the cold Earth. " The interpretation of data on 60 - and 30-year variations of the geomagnetic field result in " estimates [11] obtained to confirm the conductivity jump at the core-mantle boundary: $\sigma=10^{5}-10^{6}$ $(\mathrm{Ohm} \cdot \mathrm{m})^{-1}$ are not sufficiently reliable. 


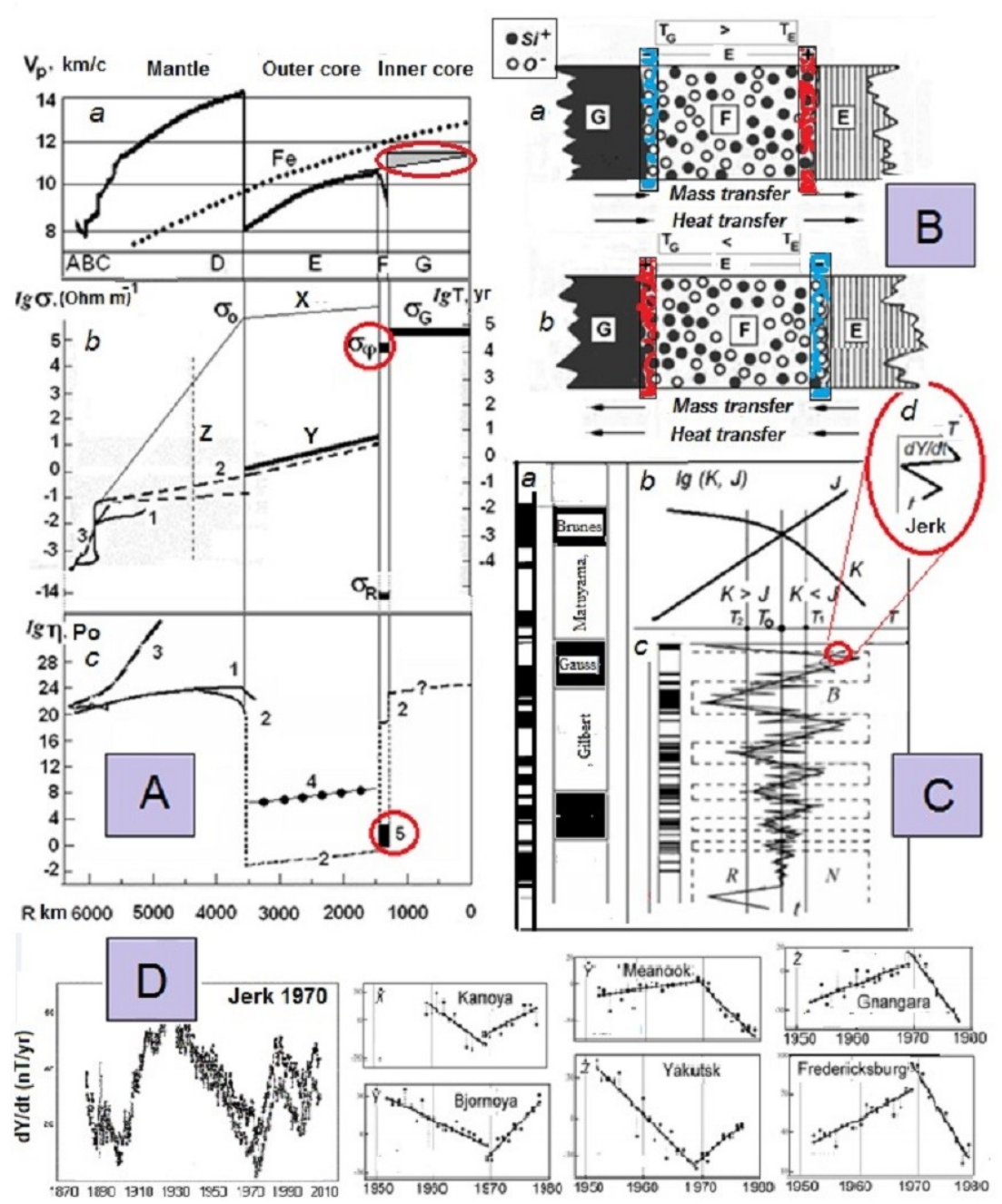

Fig. 4-A. Distribution of P-wave velocities in the Earth's core and mantle and in iron [3]- $a$.

Depth variation of the Earth's conductivity $(\sigma)$ obtained from geomagnetic variations (1), secular variations (2) and MT (3) [11] - $b$. Interpolation of the curve 3 into the area of high conductivity $\sigma_{o}, X$ - conductivity in the iron outer core, jump $\left(\sigma_{o}\right)$ at the core-mantle boundary; Y - $\sigma_{o}$ of the outer core according to our model, $\mathrm{Z}$ - the limit of the reliable values of $\sigma$. Conductivity of F - layer: $\left(\sigma_{\varphi}\right)$ geostrophic flux, $\left(\sigma_{R}\right)$ - radial transport; $\left(\sigma_{G}\right)$ - conductivity of the inner core. The right scale: $T$ diffusion time of the magnetic field.

Viscosity distribution in the Earth: 1 - under the adiabatic temperature distribution in the mantle by the Ranelli-Fisher model; 2 - accepted here; 3 - in the young Earth (before its differentiation); 4 - by seismology data [12]; 5 - viscosity of the F-layer substance (by our model)* $-c$.

Fig. 4-B. Heat and mass transfer in the F-layer: expansion cycle $(K>J)-a$, compression cycle $(J>$ $K)-b$.

Fig. 4-C. Reversal of the geomagnetic field polarity. the paleomagnetic scale - $a$, the change of condensation $K$ and evaporation $J$ rates - $b$, our model of polarity reversals - $c$, our jerk's model - $d$.

Fig. 4-D. Jerk-1970 in various regions of the Earth.

The top line $\left(2-\sigma_{o}\right)$ in Fig.4-A, $b$ shows the data on the geoelectric sections of the lower mantle [11]. Despite this result was duplicated, it is questionable because the dashed 
line B (Fig. 4-A, $b$ ) encloses the range of conductivity values corresponding to the 11 -year periods of variations. Apparently, this result is the most reliable, because variations with longer periods (tens and hundreds of years) are not regular and widespread. Therefore, the data on secular variations (curve 2) are extended by the dashed line. The conductivity jump should be observed at the intersection of the curve 2 with the core-mantle boundary to the value $\sigma=10^{5}-10^{6}(\mathrm{Ohm} \cdot \mathrm{m})^{-1}$ (line $\left.\mathrm{X}\right)$. This value corresponds to the conductivity of the iron of the outer core. Right scale Fig. 4-A, $b$ shows the period of variation related to the conductivity (on the left scale). To estimate the electrical conductivity of the core, we should have to probe it by vibrations with a period of $10^{5}$ years. Parkinson [9] argued that if any processes associated with the generation of geomagnetic field occur in the liquid core, the time of their exit to the Earth's surface should be of the same order.

However, different times follow from the practice of Geomagnetism. The minimum period of variation obtained by paleomagnetic data is about 100 years. Due to technical reasons such as a limited sedimentation rate and a small thickness of the sample a shorter period cannot be revealed. Jerks, the phenomena of modern Geomagnetism, occur over time, no longer than one year. Probably, this value should be considered the smallest period of variation of the geomagnetic field, and it corresponds to $\sigma=10^{1}(\mathrm{Ohm} \cdot \mathrm{m})^{-1}$. Extending the line 2 we get a point corresponding to the electrical conductivity $\sigma=20-30(\mathrm{Ohm} \cdot \mathrm{m})^{-1}$ at the boundary of the inner core. According to our model, in the F-layer the conductivity is (as well as in the ionosphere) tensor: $\sigma_{R}$ - across the layer, and $\sigma_{R}$ (red line) - along the layer*. As will be shown later, the value of $\sigma_{R}$ is determined by polarization manifested in the F-layer and separation of electric charges, whereas the enhanced conductivity $\sigma_{\varphi}$ of the moving Ekman layer (geostrophic wind) arises from the change of a substance properties at the critical temperature. Under critical $p T$ conditions the substance is known to have unique properties: "superfluidity" and "superconductivity*". The conductivity of the substance in the inner core $\sigma_{G}$ is the highest.

\subsection{Measurement of viscosity $\eta$ on superrotation $\Delta \omega=\left(\omega_{2}-\omega_{1}\right)$.}

To estimate the viscosity, we use the result of [12], here $I_{1}$ and $I_{2}$ are inertia moments of the mantle and the inner core, and $\omega_{1}$ and $\omega_{2}$ - their angular velocities. The rotational motion of the mantle and the solid inner core is given by the equations:

$$
I_{1} d \omega_{1} / d t=h\left(\omega_{2}-\omega_{1}\right)-M, \quad I_{2} d \omega_{2} / d t=-h\left(\omega_{2}-\omega_{1}\right),
$$

here $M$ is the moment of forces stemming from the tidal interaction in the Earth-Moon system and leading to an increase in the day duration by $1.7 \mathrm{~ms}$ for 100 years, $h\left(\omega_{2}-\omega_{1}\right)$ is the moment of friction forces governed by the liquid outer core. The outstripping rotation of the solid inner core is an obvious consequence of these equations.

The superrotation rate of the inner core relative to the mantle is estimated to be about 1 degree per year, which corresponds to the effective viscosity of the outer core $10^{3} \mathrm{~Pa} \cdot \mathrm{s}$. [13]. Constructing the model of the dipole field generation, we used even lower values of viscosity, assuming that the substance of the F-layer is in a state close to super-fluidity [14].

\subsection{Phase transition in the F-layer.}

According to the model of hot Earth condensation and evaporation or their higher stage - boiling compete in the F-layer region of the phase transition (Fig. 3-C, b). The condensation rate $K\left(\mathrm{~s}^{-1} \mathrm{~cm}^{-3}\right)$ is [15]:

$$
K \approx 10^{26} \exp \left(-3 / \ln S_{S}+\ln S_{S}\right)
$$

here $S_{S}$ - supersaturation of vapour, described by the ratio of the vapor pressure to the pressure of saturated vapour at the same temperature. The value of $S_{S}$ is determined by the temperature of the vapour, its thermodynamic potential, the size of the droplet nuclei, the surface tension of the liquid, etc. The condensation rate $K$ shows the number of elementary 
processes of particles fusion per unit time in a single volume. The value of $K$ determines the rate of the drops formation and growth.

In the mode of evaporation (boiling) mainly vapour bubbles with the rate of formation $J$ are generated:

$$
J\left(\mathrm{~s}^{-1} \mathrm{~cm}^{-3}\right)=10^{37} \exp (-W / k T)
$$

where $W$ is the energy required to form a bubble with a critical size. At a temperature $T_{o}$ (Fig 4-C, $b$ ) $K$ and $J$ become equal and the system reaches its thermodynamic equilibrium.

At a certain time $t_{o}$, the system is overheated on $\Delta T$ to a temperature of $T>T_{o}$. It is obvious that the evaporation $(J>K)$ prevails, and a part of condensed matter turns into gaseous one. The heat of the phase transition required for the formation, growth and movement of bubbles is absorbed and the system cools. In the field of formation of "high-density" bubbles there will be negative pressure and "free" space, which will be instantly filled with the surrounding matter. The volume of the Earth therewith will decrease. Then the heat will be released from the zone of the phase transition by convection in the core and possibly in the mantle. When the temperature $T$ will decrease enough for $T<T_{o}$ there will be a change of mode to the prevalence of condensation $(K>J)$. Some gaseous matter will condense with the release of the heat through a phase transition, which will result in heating of the system and an increase in its temperature. The cycle of modes changing will repeat.

\subsection{Separation of electric charges in the F-layer.}

The temperature of the processes occurring in the F-layer stabilized by the phase transition is close apparently to the critical temperature of the substance. Condensing the super-condensed and superheated vapour (supercritical liquid) substance of the inner core (let it be - $\mathrm{SiO}_{2}$ ) causes a volume increase and release of energy equal to the heat of the phase transition which induces the growth of temperature $T_{E}$.

At the boundary of the F-layer and the outer core (E) positively charged silicon ions accumulate and negatively charged oxygen ions - near the surface of the G-core. In the Flayer a double electric layer (DEL) occur. The temperature $T_{E}$ grows higher than $T_{G}$, and the substance on the surface of the E-core evaporates resulting in the absorption of the phase transition energy and reversal of the DEL polarity (Fig. 4-B $a, b$ ).

In $\mathrm{G}$-core the temperature of the substance of $T \approx(1-3) 10^{4} \mathrm{~K}$ invokes its partial ionization. The ionization degree evaluated by the Sakha formula for the constant of ionization equilibrium $k_{p}$ yields the density of electric charges [3]: $n_{e} \approx 10^{20} \mathrm{~cm}^{-3}$ (at the Gcore) and $10^{18} \mathrm{~cm}^{-3}$ (at the F-layer). In the F-layer, due to the temperature gradient and different mobility of the charges they separate giving DEL.

Using the Gibbs-Helmholtz equation for the isobaric-isothermal process, it is possible to estimate the EMF of DEL. Depending on condensation conditions the derivative $d T / d p$ like EMF can change its sign.

A possible mechanism that causes the separation of charges when they move along the F-layer is thermodiffusion, during which heavy particles (here - positive ions) move along the heat flow, light ones (negative ions, electrons) - against the flow.

\subsection{Features of the electric field of DEL.}

According to our model, the polarity of the DEL governed by the direction of heatand-mass transfer, reverses when it changes and the radial electric field $E_{R}$ the value of which would not be infinitely large appears in the F-layer (Fig.4-B). For example, in the atmosphere, the field $E \sim 10^{4}-10^{5} \mathrm{~V} / \mathrm{m}$ is formed due to the separation of electric charges in thunderclouds.

Estimating the intensity of the electric field $E_{R}$ in the F-layer: $E_{R}=q /(C \times \Delta R)$, here $q=$ $\mathrm{NeV}$ - electrical charge of the layer, $\Delta R$ - its thickness, $N$ - concentration of charges, $e$ is the electron charge, $V$ is the volume of the spherical "capacitor": $V=4 \pi R^{2}{ }_{\mathrm{G}} \Delta R$, where $R_{G}$ 
is the radius of the inner core, and $C$ - the electric capacity of DEL: $C=4 \pi \varepsilon_{o} R^{2}{ }_{\mathrm{G}} / \Delta R, \varepsilon_{o}-$ the electric constant, we get: $E_{R}=\Delta R N e / \varepsilon_{o}$ After substituting $E_{R}=10^{-3} N$. Let $E_{R} \leq 10^{8}$ $\mathrm{V} / \mathrm{m}$ then $N \leq 10^{11} \mathrm{~m}^{-3}\left(N=10^{3} E_{R}\right)$ which is much lower than that we see $\left(N \approx 10^{20} \mathrm{~m}^{-3}\right)$ [3] and the separation mechanism we discuss is valid.

What is DEL? It is a set of two surfaces $S$ and $S$ ' close to each other and oppositely charged, where the charge densities $n$ and $n$ ' of opposite elements at both surfaces are equal in magnitude and opposite in sign [16]. The distance between the surfaces is smaller than the distance from them to the considered points of the field. The potential of DEL at some point $\mathrm{P}$ is [16]:

$$
\varphi=-\int_{S} \tau k \operatorname{grad}\left(\frac{1}{R}\right) d S .
$$

$\tau=n l$, where $l$ is the thickness of the layer (the distance between $S$ and $S^{\prime}$ ), $k$ is the direction of the normal to DEL, $R$ is the distance from $S$ to the point P. The value of $\tau$ corresponds to the power (moment) of DEL.

There is a jump of $4 \pi \tau$ of DEL potential directed from the negative side of the layer to the positive one. DEL is surface of discontinuity of the of the potential continuity: $\varphi_{2}-\varphi_{1}=$ $4 \pi \tau$. The electric field on the surface of the potential discontinuity goes to infinity, i.e., loses physical meaning. DEL charge and power would rise while as the potential jump $\varphi_{2}$ $\varphi_{1}$ becomes equal to the potential difference applied externally. The current through the DEL will stop $(j=0)$ and the field strength $E \rightarrow 0$ (Fig. 4-B), i.e. the electrostatic equilibrium will be achieved. Thus, DEL provides polarization of the polarization of the Flayer boundaries. As it follows from the model of DEL the electric charges are located near the boundaries of the $\mathrm{F}$-layer occupying the thickness $\delta$ and the height $A$ of the layer (Fig. 4-B). Like the dielectric the electrical conductivity of the F-layer along the radius is very small: $\sigma_{R} \sim j_{R} / E_{R}$ (Fig. 4-A, b).

\subsection{Daily rotation of the F-layer and the appearance of the initial geomagnetic field.}

Is the emergence of the magnetic field possible through to the daily rotation of electric charges? Janowski in his well-known book (reference at [3]) writes that this idea "contradicts the principle of relativity, because rotating together with the charges the observer on the surface of the Earth remains motionless relative to them." The generation of the magnetic field through the rotation of electric charges was experimentally proved by Eichenwald (reference at [3]) who measured the magnetic field caused by the rotation of a polarized dielectric.

The magnetic field of the rotating DEL can be represented as the field of the current $I$ over the outer surface of the F-layer (at the radius $R_{F}$ ): $B \approx \mu I_{F} / R_{F} . \quad I$ is equal to the corresponding current density $j$ multiplied by the size of the surface section $S, j=N e \omega 2 \pi R_{F}$ $=100 \cdot 10^{-19} N$. If $N \approx 10^{11} \mathrm{~m}^{-3}$, then $j=10^{-6} \mathrm{~A} / \mathrm{m}^{2}$. The transfer area of the rotating charge $S$ we evaluated earlier [3, 4]: $S \approx \delta A=10^{9} \mathrm{~m}^{2}$. Then the current $I_{\mathrm{o}}=10^{3}$ A. The magnetic field of rotating charges:

$$
B_{\mathrm{o}}=\mu I_{\mathrm{o}} / R_{G}=4 \pi 10^{-7} \cdot 10^{3} / 10^{6}=10^{-9} \mathrm{~T} .
$$

To obtain the value of the field $B \approx 3 \cdot 10^{-3} \mathrm{~T}$, it is necessary to increase $B_{\mathrm{o}}$ in $k_{B}=B / B_{\mathrm{o}}>10^{7}$ times.

\section{The formation of the main EMF. 4.1. Current systems at the F-layer.}

The current systems in the layer with thickness $A$ between the inner $\mathrm{G}$ - core and the outer E-core are shown in Fig. 5. 


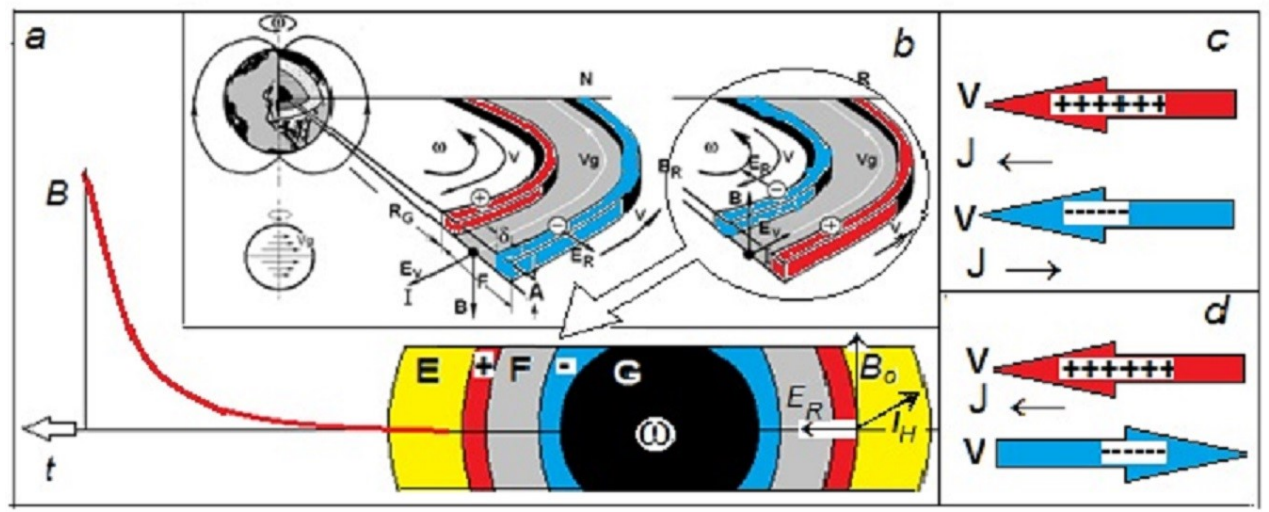

Fig. 5. Strengthening of the field $B$ at the expense of the Hall current $I_{H}$ in radial electric field $E_{R}-a$, current scheme at the F-layer for direct $(\mathrm{N})$ and reversed $(\mathrm{R})$ polarity of EMF - $b$, current $J$, velocities of the charges movement $V-\mathrm{c}, d$.

The hydrodynamic transfer of the F-layer in boundary $\delta$ - layers is opposite directed with a speed $v$. Between $\delta$ - layers there is a geostrophic thermal wind, like to that in the atmosphere. When the polarity of the geomagnetic field reverses, the sign of the charge carrier in $\delta$-layers changes resulting in a change of the currents direction and, accordingly, of the direction of the field vector $B$.

\subsection{The characteristic time of change of the field module.}

As known the variation of EMF vector parameters during the field excursions is faster than this of the field intensity from its fall up to restoring of the initial magnitude.

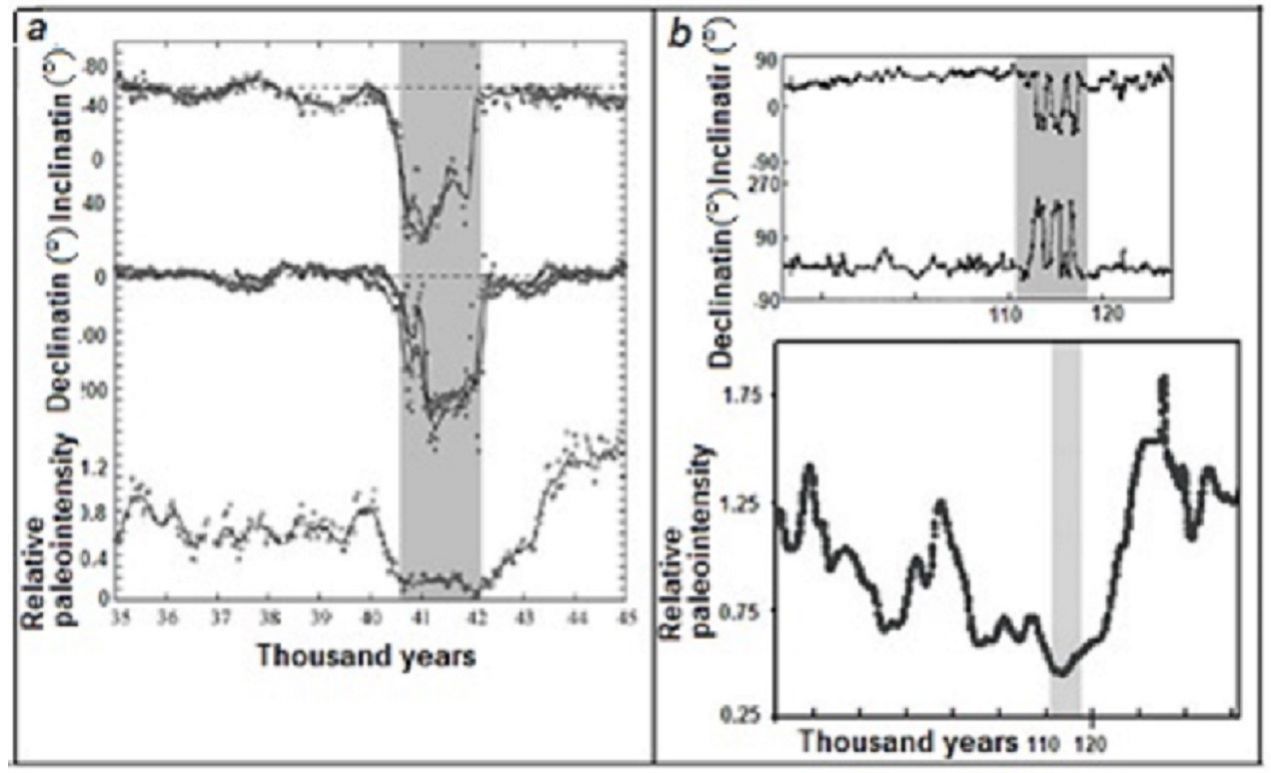

Fig. 6. Characteristics of Laschamp (a) and Blake $(b)$ excursions. Grey vertical bands show the variation time of the EMF vector parameters [17].

Let's use the fundamental equation of magnetic hydrodynamics:

$$
\partial B / \partial t=\operatorname{rot}(\mathrm{u} \times \mathrm{B})+\eta \nabla^{2} \mathrm{~B}=v B / L+\eta_{M} B / L^{2}
$$


where $\eta_{M}=(\mu \sigma)^{-1}$ is the magnetic viscosity, an analogue of the kinematic viscosity $v$. The Reynolds number defines the boundary between laminarity and turbulence of the flow: Re $=L v / \eta$, here $L$ and $v$ are characteristic length and rate of the flow. By analogy, the magnetic Reynolds number Rem can be written as: $R e m=L v / \eta_{M}$.

The value of $\partial B / \partial t$ can be estimated as by the rate of decrease of the geomagnetic field module nowadays: $\partial B / \partial t \approx 10 \mathrm{nT} /$ year, so from the field behavior at the time of reversals and excursions, when the field drops practically to zero and then it restores its previous level in about 100 years $\left( \pm 50\right.$ years) $\partial B / \partial t \approx 600 \mathrm{nT} /$ year. Assuming $L=2 \pi R_{F}=$ $6000 \mathrm{~km}$, we can estimate the flow rate $v$, the values of $\eta_{M}$ and Rem. Since $v B / L>>\eta_{M}$ $B / L^{2}$, the rate of hydrodynamic transport: $v=\partial B / \partial t(L / B)$,

- in the quiet period: $v \approx 10^{-4} \mathrm{~m} / \mathrm{s}$. If the electrical conductivity of the matter in the F-layer $\sigma=10^{4}-10^{5}, \eta_{M} \approx 10-100 \mathrm{~m}^{2} \mathrm{~s}^{-1}$, Rem $\approx 100$.

- during reversals: $v \approx 10^{-2} \mathrm{M} / \mathrm{c}$, a $R e m \approx 10^{3}$. For comparison, the Reynolds number $R e=$ $L v / \eta=600 v \approx 6$. The estimates show that the character of the flow in both cases is laminar. It should be noted that estimations of $\partial B / \partial t$ are related rather to the variation time of the EMF vector parameters (Fig. 6, grey bands) than to real.

The real values of the field in the region of the F-layer and, probably, the rate of its change $\partial B / \partial t$ are about 100 times greater, but they do not affect the value of $v \sim \partial B / B$.

By our model the flow rate in the field mode of generation-amplification: $v \approx 10^{-2}$ $\mathrm{m} / \mathrm{s}$, and in its mode of a stable state: $v \approx 10^{-4} \mathrm{~m} / \mathrm{s}$. It remains unclear whether a flow with such speeds is possible in the F-layer? Notice, for comparison, that the so-called Western drift and super-rotation of the inner core are hydrodynamic opposite directed flows with approximately equal velocities of the order of $0.3^{\circ}$ per year, which corresponds to a transport velocity of $v_{g} \approx 2 \cdot 10^{-4} \mathrm{~m} / \mathrm{s}$.

\subsection{The effect of amplification of the initial field.}

Let's estimate if the field amplification due to its interaction with the conducting medium moving with velocity $v$ is available. The field $B$ after its $k$ times amplification is: $B$ $=\mu I \Delta R / R^{2}{ }_{G}$ similarly to $B_{\mathrm{o}}=\mu I_{\mathrm{o}} \Delta R / R^{2}{ }_{G}$. Since all other parameters except currents do not change and $I=j S$, where the cross-sections of the currents are constant, then the amplification effect comprises $k=j / j o$. The density of the current of rotating charges: $j_{o}=$ $n_{\mathrm{e}} e \omega R_{\mathrm{F}}$; the density of the Hall current $j$ follows from the formula of the Hall EMF: $E \varphi=$ $1 / e n_{\mathrm{e}}(j \times B)$. Then the coefficient of the field amplification due to the Hall dynamo effect is: $k=E_{\varphi} / B_{0} \omega R_{\mathrm{F}} \approx 10^{9} E_{\varphi . .}$

This coefficient estimated above is: $k_{B}=B / B_{0} \sim 10^{7}$ times and $E \varphi=10^{-2} \mathrm{~V} / \mathrm{m}$.

There are three ways to estimate $E_{\varphi}$ :

1. $E_{\varphi}=j / \sigma_{\varphi}=I / S \sigma_{\varphi}=2.5 \cdot 10^{8} / 10^{9} \times 10^{5}=2.5 \cdot 10^{-6} \mathrm{~W} / \mathrm{m}$;

2. $E_{\varphi}=P / I L=10^{12} / 10^{10} \times 6 \cdot 10^{6}=1.5 \cdot 10^{-5} \mathrm{~W} / \mathrm{m}$;

3. $E_{\varphi}=\mathrm{v} B=10^{-2} \times 3 \cdot 10^{-4}=3 \cdot 10^{-6} \mathrm{~W} / \mathrm{m}$.

As we see the value of $E \varphi$ obtained from the coefficient of amplification is 3 to 4 orders higher than other estimates. The fact is that we assumed this coefficient as a one-time (per one rotation of the "current ring") field amplification from $B_{\mathrm{o}}$ to $B$. The time of one rotation is $t=L / v=20$ years.

The coincidence of $E$-values will be valid if the intensity of the geomagnetic field is two orders less than the value of $P=10^{12} \mathrm{~W}$ accepted. The intensity we discuss here is a product of current-to-voltage developed by the hydrodynamic interaction of a moving flow with a magnetic field, where $E_{\varphi} I L$ is the voltage. The efficiency of the Earth's energy converting into a magnetic field is possible to be significantly less than $1 \%$.

The dissipation power of the geomagnetic field was estimated [6, p. 166] to be: $10^{12}$ $\mathrm{W}$ by Braginsky, $2 \cdot 10^{9} \mathrm{~W}$ by Bullard, $2.3 \cdot 10^{10} \mathrm{~W}$ by Malkus. Rochester assumes its lower value as $10^{9} \mathrm{~W}$ and the upper one as $4 \cdot 10^{13} \mathrm{~W}$. Thus, our estimate does not leave this 
interval. For comparison, the Earth thermal capacity measured by the heat flux is equal to $4 \cdot 10^{13} \mathrm{~W}$. Thus, the generation of the Earth's magnetic field accounts for less than $0.1 \%$ of the total energy. Stored in the inner core the energy is consumed through the PT 1-st kind in the F-layer. The obvious consequence of our model involves the disappearance of the Earth's magnetic field after all matter of the inner core would exhaust. We would like to note that the Hall dynamo at the F-layer to a great extent repeats the known model of ionospheric dynamo [18].

\subsection{Dynamo of the F-layer.}

The ionospheric dynamo is agreed to be excited in the conducting layer of the ionosphere, firstly, due to the wind transport of the conductive medium across the Earth's magnetic field and, secondly, due to the tensor nature of the conductivity. In our model, the magnetic field is the Earth's field. Dynamo in the F-layer as the generation model needs the initial seed field which we assume to be the separation of charges and their daily rotation. Then the radial electric field $E_{R}$ which polarity change leads to the field reversal becomes obvious in the scheme of dynamo generation. This means that, unlike the first scheme, the boundary layer should change the direction of the wind to the opposite one. It follows that rather other currents that are clearly associated with the PT processes occurring in the Flayer than geostrophic winds take part in the generation of the field. Then now on the outer surface of the F-layer the wind is Western directed, i.e. the medium moves to the West. Naturally, at modern polarity the current on the inner surface of the F-layer has an Eastern direction (as in the ionosphere). Let's pay attention to the analogy: currents are flowing in the Eastern direction near the positively charged surface (ionosphere and G-core), and, on the contrary, in the Western, if the surface has a negative charge (land and E-core). Apparently, the reason is that when the polarity of the geomagnetic field reverses, the direction of radial mass transfer in the PT region changes, which, with the same direction of the Earth rotation (due to Coriolis forces), leads to a change in the direction of horizontal transfer. In addition to this feature, which does not affect the processes by the first scheme, both models are identical.

\subsection{Superrotation of the G-core and the westward drift of the FSV.}

Tidal deceleration, which is most effective in the upper shells of the Earth, and the presence of a liquid outer core determine the fundamental possibility for superrotation of the inner core. The hypothesis of the differential rotation of the molten substance in the external iron core was used earlier to construct a model of the geomagnetic field generation with the dynamo mechanism and to explain such a phenomenon in Geomagnetism as Western drift.
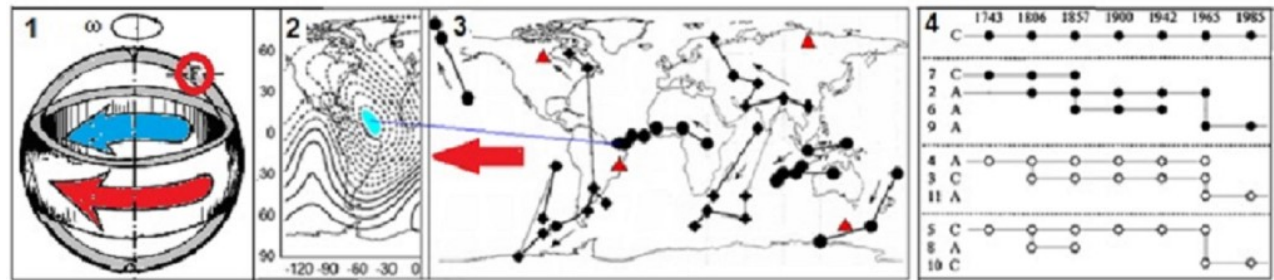

Fig. 7: The surfaces of the F-layer, a blue arrow - superrotation of the G-core, a red arrow - the drift of the secular variation focuses (FSV) - 1. Brazilian FSV - 2. The map of FSV, right - the map of the FSV drift from 1743 to 1985 , triangles - GMA. Numbers of FSV are shown at the scheme. The red arrow is the direction of the Western drift - 3. A scheme. The evolution of secular variation focuses (C-cyclones; A-anticyclones) - 4.

The superrotation of the inner core was confirmed applying the data on the splitting in the spectra of the Earth's natural oscillations in the inner core and their possible variation 
in time [14]. As shown the reason for the Western drift is the implementation of the law of conservation of the impulse momentum in the system. Velocities coincidence of the superrotation and the Western drift is not random.

5. The North magnetic pole moves steadily according to our model of its drift.

(The paper was submitted to Bulletin of KRAESC. Physical \& Mathematical Sciences).

6. Two new problems of Geomagnetism: 1) when will the geomagnetic field disappear [19], Fig. 8, 9 and 2) why the mode of the P- and S-waves velocities is curious [14, 3]. Fig. 4-A $(\mathrm{a}, \mathrm{b}, \mathrm{c})$ - areas are outlined by red.

\section{Conclusions}

A fundamentally new model of generation and evolution of the Earth's magnetic field (EMF), planets and large satellites is proposed.

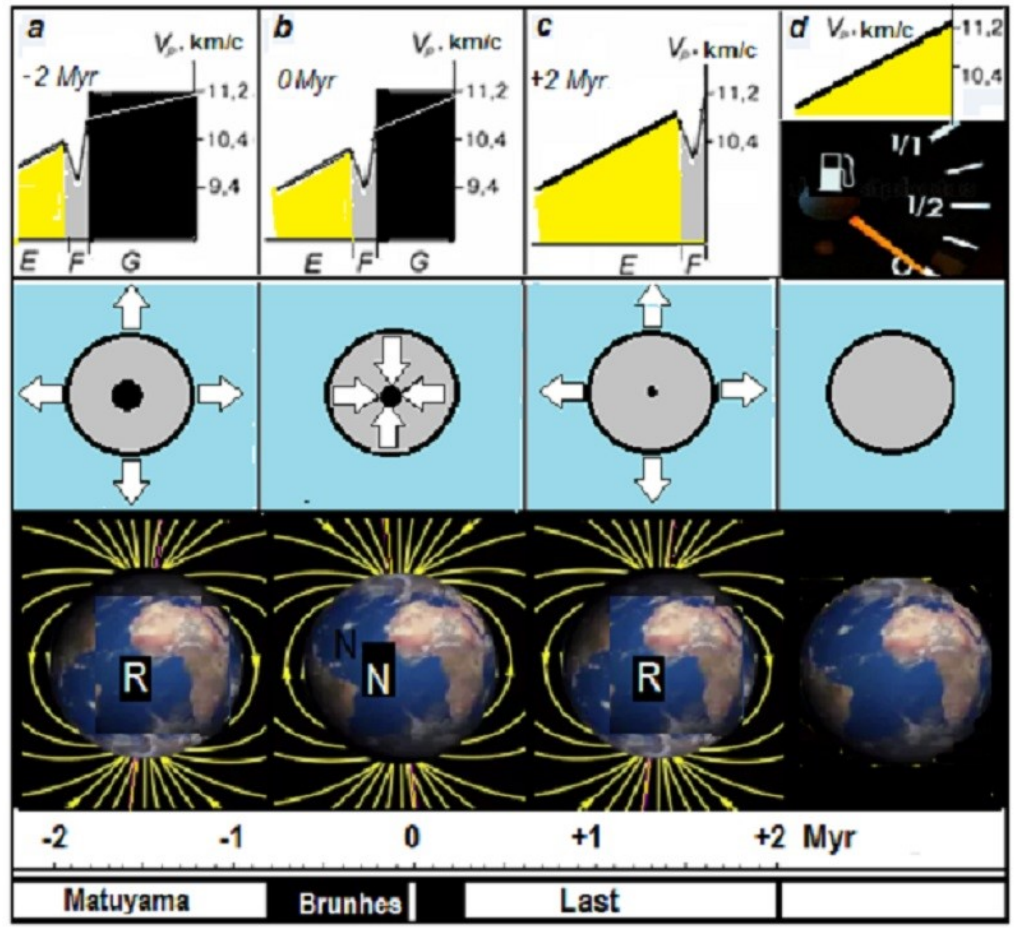

Fig.8. Evolution of the inner core: During the Matuyama period the Earth has been expanding and the EMF had the reversed sign - $a$. Nowadays (Brunhes epoch) the Earth is shrinking, the polarity of the EMF is normal - $b$. In the era following the modern one (Last), the Earth will be expanding until the complete disappearance of the G-core - c. G - core disappeared ("gas tank" is empty), there is no $\mathrm{EMF}-d$.

In contrast to the commonly used kinematic dynamo as a scheme of the EMF generation, the new model is based on the author's model of hot expanding Earth, using the features of this idea. The main advantage of both the hot Earth model and the EMF generation model is that the hot Earth provides an obvious energy source, it is the phase transition (PT) "condensation-evaporation". PT of the "hot Earth" occurs at a high temperature $T \approx 30000 \mathrm{~K}$.

At this temperature the substance is in a state of superheated steam, possibly in the state of astrophysical plasma. It is compressed by self-gravitation to a volume equal to that of the outer core. The density of such a substance is about $35 \mathrm{~g} / \mathrm{cm}^{3}$. During its 
evolution, the substance of the Earth cools and condenses and then it crystallizes with further decrease of temperature. Thus the volume of the Earth matter increases approximately in 2-3 times. The Earth is expanding during the condensation period, and it is shrinking when it cools down and evaporation occurs. Hence the first mode is dominating the Earth is expanding.

At a temperature of $30000 \mathrm{~K}$ the substance is thermionized (here we accept that it is $\mathrm{SiO}_{2}$ ) and positive and negative ions are formed. $P T$ is characterized by heat and mass transfer, resulting in charges separation, the appearance of a radial electric field $E_{R}$ and the formation of a double electric layer (DEL). The daily rotation of the DEL leads to the generation of a weak dipole magnetic field $B_{o}$ directed along the axis of the planet rotation. These components lead to the emergence of the Hall EMF and the Hall current which causing the generation of the EMF is an amplifier of field $B_{o}$.

Reversing of the mode of PT (condensation - evaporation) to the mode (evaporation-condensation) changes the direction of heat and mass transfer and the polarity of $E_{R}$ and DEL that cause a change in the direction of the field $B_{o}$, the direction of the Hall current and the polarity of the EMF, otherwise, the field reversal. Condensation mode leads to cooling of the system, evaporation one - to its heating. The cycle repeats. The heat of $\mathrm{SiO}_{2}$ condensation (evaporation) is $15 \mathrm{~kJ} / \mathrm{g}$ that determines the source energy.

The location of the energy source obtained by several methods points to the energy source of the Earth located in the F-layer between the inner and outer cores. To date, this source has consumed approximately $99 \%$ of its energy.

Superrotation of the inner core with a speed of about of 0.5 degree/year causes an East directed impulse over the surface of the F-layer. By the law of momentum conservation on the other surface of this layer, at its border with the outer core, there is the movement in the Western direction. This thin layer in which ions arising from the mass transfer in the PT are concentrated is the so-called layer of Western transfer widely discussed in Geomagnetism, which occurs at the same rate as superrotation. This layer implements global magnetic anomalies (GMA), the focuses of secular variation (FSV), Hall current flows.

By the model proposed the GMA is a Rossby vortex like the long-lived vortices in the atmospheres of Jupiter and Saturn. Rotating the GMA vortex captures ions of the surface layer, that leads to the generation of the magnetic field. By our approach it possible to present the EMF as a set of axial dipole and four radial dipoles which are GMA. Moreover, the modules of the GMA field are changing their value, and their value itself is commensurate with the field of the main dipole.

The proposed model of the EMF allows to explain almost all the features of Geomagnetism. In particular, the model allows to estimate the time the EMF will exist (Fig. 8). The disappearance of the MF at the Moon and Mars, the MF of Mercury, etc. follows from our model too.

Our model provides a simple and unambiguous solution to such global problems of the Earth's magnetism as reversals, the correlation between the frequency of reversals and the tectonic intensity, the famous paradox "superchron-superplume" and to the main problem of Geomagnetism - what is the source of the field generation.

\section{References}

1. P. Zhang, R. Cohen, and K. Haule, Nature, 517, 605-607 (2015)

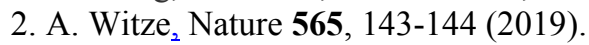

3. V.V. Kuznetsov, Introduction to the physics of the hot Earth (KamGU, PetropavlovskKamchatsky, 2008) (in Russian)

4. V.V. Kuznetsov, Physics of the Earth and the Sun system (Models of formation and evolution) (IGG SB RAS, Novosibirsk, 1984) (in Russian) 
5 V. A. Magnitsky, Internal Structure and Physics of the Earth (Nedra, Moscow, 1965) (in Russian)

6. R Meservey, Science, 166, 609-611 (1969)

7. K Vogel, Recent crystal movements in the light of Earth expansion theory. $6^{\text {th }}$ International Symposium "Geodesy and Physics of the Earth". Potsdam. 284-289 (1989)

8. V V Kuznetsov, Vestnik KRAUNC. Phys.-Mat. Science, 2(9), 36 (2014)

9. W. D. Parkinson, Introduction to Geomagnetism (Scottish Ac. Press, Edinburg and London, 1983)

10. L. D. Landau, E. M. Lifshitz, Hydrodynamics (Moscow, Nauka, 1986) (in Russian) 11. N. M. Rotanova, A. P. Pushkov, Deep electrical conductivity of the Earth (Moscow, Nauka, 1982)

12. G.G. Denisov, V.V. Novikov, Dokl. Ross. Nauk, 362, 484 (1998) (in Russian)

13. B G Bills, J. Geoph. Res., 104 (B2), 2653(1999)

14. V.V. Kuznetsov, Phys. Usp. 40, 951 (1997) (in Russian)

15. Ju.I. Frenkel, Kinetic theory of liquids (Leningrad, Nauka, 1975) (in Russian)

16. I. E Tamm, Fundamentals of theory of electricity (Moscow, Nauka, 1966) (in Russian)

17. N.D. Kuznetsova, V.V. Kuznetsov, Vestnik TGU, N354, 199-204 (2012) (in Russian)

18. H. Rishdath, O.K. Garriott, Introduction to ionospheric physics (A.Pr. NY \& London, 1969)

19. V. V. Kuznetsov, Geologiya i MSR Sibiri, 3(19), 89-98 (2014) (in Russian) 